\title{
AMEGO: Dark Matter (ICRC2017)
}

\author{
Regina Caputo* \\ UMD/NASA/GSFC \\ E-mail: regina.caputo@nasa.gov
}

\section{Manuel Meyer ${ }^{a}$, Miguel A. Sánchez-Conde ${ }^{b}$}

${ }^{a}$ W. W. Hansen Experimental Physics Laboratory, Kavli Institute for Particle Astrophysics and Cosmology, Department of Physics and SLAC National Accelerator Laboratory, Stanford University, Stanford, California 94305, USA

${ }^{b}$ Instituto de Física Teórica UAM/CSIC and Departamento de Física Teórica, Universidad Autónoma de Madrid, E-28049 Madrid, Spain

\section{The AMEGO Team}

https://asd.gsfc.nasa.gov/amego/

\begin{abstract}
The era of precision cosmology has revealed that $\sim 85 \%$ of the matter in the universe is dark matter. Two leading candidates, are weakly interacting massive particles (WIMPs) and weakly interacting sub-eV particles (WISPs) like axions and axionlike particles. Both WIMPs and WISPs possess distinct $\gamma$-ray signatures. Data from the Fermi Large Area Telescope (Fermi-LAT) continues to be an integral part of the search for these dark matter signatures spanning the $50 \mathrm{MeV}$ to $>300 \mathrm{GeV}$ energy range in a variety of astrophysical targets. Thus far, there are no conclusive detections; yet, there is an intriguing excess of $\gamma$ rays associated with the Galactic center that could be explained with WIMP annihilation. The angular resolution of the LAT at lower energies makes source selection challenging and the true nature of the detected signal remains unknown. WISP searches using, e.g. supernova explosions, spectra of blazars, or strongly magnetized environments, would also greatly benefit from increased angular and energy resolution, as well as from polarization measurements. To address these, we are developing AMEGO, the All-sky Medium Energy Gamma-ray Observatory. This instrument has a projected energy and angular resolution that will increase sensitivity by a factor of 20-50 over previous instruments. This will allow us to explore new areas of dark matter parameter space and provide unprecedented access to its particle nature.
\end{abstract}

35th International Cosmic Ray Conference - ICRC2017

10-20 July, 2017

Bexco, Busan, Korea

${ }^{*}$ Speaker. 


\section{AMEGO}

The All-Sky Medium Energy Gamma-ray Observatory (AMEGO) is a mission in consideration as a probe for the 2020 Astrophysics Decadal review. ${ }^{1}$ AMEGO will survey the entire sky every 3 hours and observe $80 \%$ of the sky every orbit with its wide field of view $(\sim 2.5 \mathrm{sr})$ and excellent continuum sensitivity between $200 \mathrm{keV}$ and above $10 \mathrm{GeV}$ (see Figure 1). The AMEGO design uses well understood, well tested technologies with significant space heritage. Figure 1 is a diagram of the instrument. For more details please see Ref. [1].
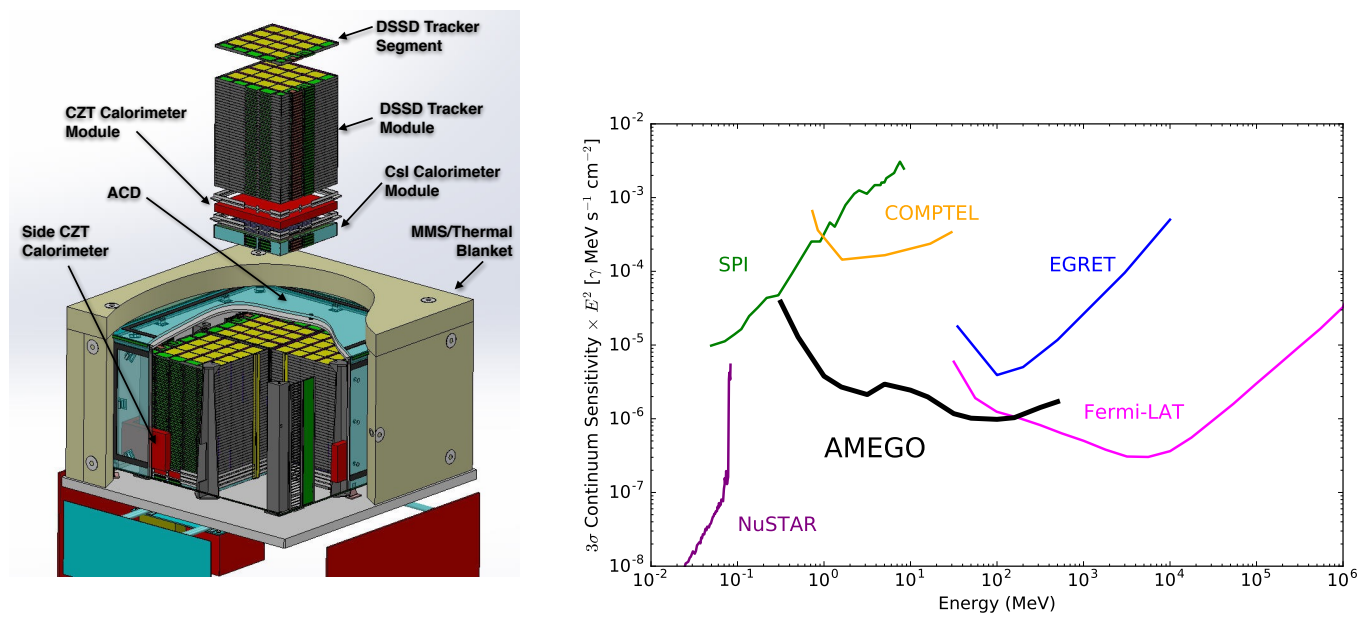

Figure 1: (left) AMEGO hardware design [1]. (right) AMEGO integral flux point source sensitivity between $\sim 200 \mathrm{keV}$ and over $10 \mathrm{GeV}$ corresponding to a $3 \sigma$ detection in 3 years. (Note: Energies up to $1 \mathrm{GeV}$ were simulated; however, the instrument is sensitive above this energy). Also shown in comparison are the sensitivities of previous or current space missions in a similar or complementary energy range.

\section{Science Case}

The AMEGO energy band represents the transition between the thermal and non-thermal Universe. It is the only part of the electromagnetic spectrum where it is possible to directly observe nuclear processes (atomic nuclei de-excitations and excitations). Specifically, it covers the positron annihilation line at $511 \mathrm{keV}$. Also, large populations of known sources exist with their peak power output in the $\mathrm{MeV}$ range, making its study of extreme relevance to understand source energetics. The AMEGO mission will initiate breakthroughs in our understanding of extreme environments:

- Astrophysical Jets: understand their formation and evolution, their acceleration mechanisms.

- Compact Objects: identify the physical processes in the most extreme conditions.

- MeV Spectroscopy: measure the properties of element formation in dynamic systems.

\footnotetext{
${ }^{1}$ https://pcos.gsfc.nasa.gov/physpag/probe/probewp.php
} 
- Dark Matter: test models that predict dark matter signals in the MeV band.

These physical properties exist in an array of objects including pulsars and magnetars, $\gamma$-ray bursts and multi-messanger astrophysics [2], active galaxies [3], and dark matter.

Dark matter is one of the greatest outstanding mysteries that spans particle physics, astrophysics and cosmology. Data from the Fermi Large Area Telescope (LAT) have been used to explore well motivated dark matter candidates such as weakly interacting massive particles (WIMPs) and weakly interacting sub-eV particles (WISPs) like axions and axionlike particles. As groundbased air Cherenkov telescopes push to higher energies, the $\mathrm{MeV}$ regime remains largely unexplored. In the following sections, we will discuss the possibilities for both WIMP (Section 3) and WISP (Section 4) searches with AMEGO.

\section{WIMP searches with AMEGO}

WIMPs are a hypothetical class of particles that could constitute dark matter. They interact via gravity and any force (or forces) which is as weak or weaker than the weak nuclear force. All WIMPs must have been produced thermally in the early Universe, as have all particles of the standard model according to Big Bang cosmology [4]. To be consistent with observations of the cosmic microwave background (CMB) and large scale structure, WIMPs must not travel relativistically (i.e. they should be "cold" instead of "hot"). Obtaining the correct abundance of dark matter today via thermal production requires a self-annihilation cross section of $\langle\sigma v\rangle \simeq$ $3 \times 10^{-26} \mathrm{~cm}^{3} \mathrm{~s}^{-1}$, which is consistent with what is expected for a new particle of mass $\sim 100 \mathrm{GeV}$ that interacts via the electroweak force (see Ref. [4] and references therein). A WIMP annihilating via a a force weaker than the weak nuclear force could subsequently have different masses and still produce the correct relic abundance.

Dark matter searches using data from the Fermi-LAT were the first method of indirect detection to be sensitive enough to test thermal relic cross sections for WIMP annihilation for masses between $\sim 500 \mathrm{MeV}$ and $\sim 100 \mathrm{GeV}$. Figure 2 is a summary of dark matter searches with the Fermi-LAT. The best objects to search for WIMP annihilation are those with low astrophysical $\gamma$-ray backgrounds and yet a sufficiently large " $J$-factor" for the $\gamma$-ray signal to be detected with the instrument. ${ }^{2}$ Stellar kinematic data (i.e., velocity dispersion profiles) indicate that the dwarf spheroidal satellite galaxies (dSphs) of the Milky Way contain a substantial dark matter component [5]. As of now, however, there is no evidence of dark matter annihilation from dSphs, thus putting into question the thermal relic WIMP in the mass range from $500 \mathrm{MeV}$ to $100 \mathrm{GeV}$ [6]. The largest uncertainty in these constraints comes from the precise determination of the astrophysical $J$-factor.

The single brightest source of WIMP dark matter annihilation visible from Earth would be the center of the Milky Way [7]. It has a $J$-factor at least two orders of magnitude larger than that of dSphs; yet, the dark matter density distribution in this region is very uncertain [8]. Despite the lack of a WIMP annihilation signal from dSphs, there is an intriguing excess at the center of the Milky Way (GCE). The spatial and spectral distributions of the GCE match that of a WIMP-like dark matter particle with a mass between 20 and $50 \mathrm{GeV}$ and $\langle\sigma v\rangle$ at the thermal relic (see Ref. [9] and references therein, and Fig. 2). However the spectrum is also consistent with a population of

\footnotetext{
${ }^{2}$ The " $J$-factor" is the integral along the line of sight of the density of the dark matter squared.
} 
pulsars at the very center of the galaxy (see Ref. [10] and references therein). The limitations of the LAT data in this regime are due in part to the worsening angular resolution at lower energies $(\leq 1 \mathrm{GeV})$. Also, the spectral energy distributions of over $50 \%$ of the $\gamma$-ray sources peak in the $\mathrm{MeV}$ regime, thus data from AMEGO will be critical to disentangle a population of point sources residing at the center of the Galaxy from a dark matter origin of the GCE. This will ultimately lead to an improved detection sensitivity to dark matter annihilation from the Galactic center using both Fermi-LAT and AMEGO observations.

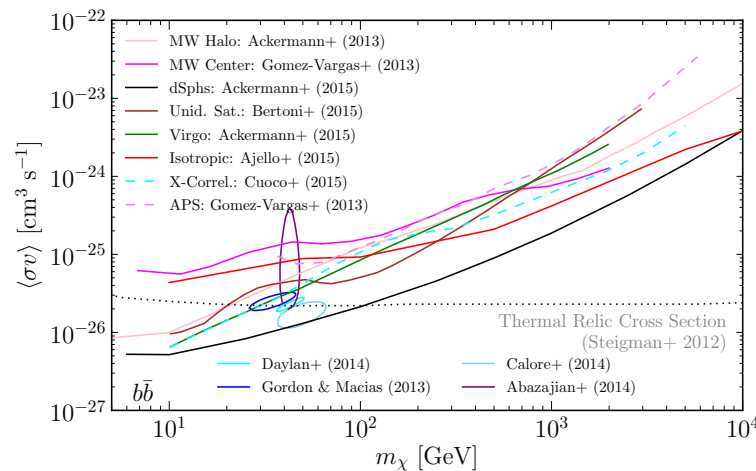

Figure 2: Summary of main dark matter results obtrophysical targets (see legend). Lines refer to upper limits on the annihilation cross section while closed contours correspond to the GCE as due to dark matter annihilations. From Ref. [11].

In general, dark matter with any mass from $\sim \mathrm{MeV}$ to tens of $\mathrm{TeV}$ can achieve the correct relic abundance by annihilating directly into standard model particles [12]. Yet, the lower part of this mass range can not be fully explored using existing instruments. Direct dark matter detection experiments, which search for signals of dark matter scattering off nuclei, lose sensitivity to a dark matter particle which is lighter than a few GeV. Collider-based searches for dark matter are also, generally, insensitive to lower masses [13]. Interestingly, limits on dark matter annihilation from the CMB do not exclude many scenarios at low masses, either [12]. To probe the universe for MeVscale dark matter, AMEGO is needed.

One class of $\mathrm{MeV}$ scale dark matter is an electromagnetic-like mediator for dark matter, i.e. a dark photon ( $\left.\mathrm{A}^{\prime}\right)$. The dark photon is a massive hidden sector particle which would couple to both dark matter and to the standard model (see Ref. [14] and references therein). Dark photons would be detectable by mixing with ordinary photons, producing features in the electromagnetic spectrum near the mass of $\mathrm{A}^{\prime}$. The dark photon would also interact with known particles and therefore produce an enhanced flux of $\gamma$ rays at around an order of magnitude lower energy than their mass [15].

Progress in searches for MeV mass dark matter is accelerating. Several proposed experiments which focus on $\mathrm{MeV}$ dark matter (such as Belle II, fixed target experiments and direct detection experiments) are in their infancy [14]. The time is right for a complementary telescope like AMEGO, to observe the sky in the MeV energy band.

\section{WISP searches with AMEGO}

As an alternative to WIMPs, dark matter could be made up entirely of WISPs that have masses in the sub-eV range and would be non-thermally produced in the early Universe (see e.g. Ref. [16] for a review). One of the most well motivated hypothetical particles that falls in this category is the axion $[17,18,19]$, a by-product of the Peccei-Quinn mechanism proposed to solve the so-called strong CP problem in QCD, but soon realized to be also a viable dark matter candidate $[20,21,22]$. 
WISPs could be detected through an oscillation to photons in external magnetic fields [23]. For the QCD axion, its mass $m_{a}$ and photon coupling $g_{a \gamma}$ are proportional, ${ }^{3}$ whereas this is not the case for general WISPs such as axionlike particles (ALPs). In general, the photon-WISP oscillation could lead to three observables: (1) spectral features around a specific energy (2), a photon flux from sources for which otherwise no emission should be detected, and (3) a change of the photon polarization. The energy at which the spectral features should occur in the case of axions or ALPs is $E_{\text {crit }} \sim 2.5 \mathrm{GeV}\left|m_{\mathrm{neV}}^{2}-\omega_{\mathrm{pl}, \mathrm{neV}}^{2}\right| / g_{11} B_{\mu \mathrm{G}}$ (e.g. Ref. [25]), with $m_{\mathrm{neV}}=m_{a} / \mathrm{neV}, \omega_{\mathrm{pl}, \mathrm{neV}}$ the plasma frequency of the medium in neV, $g_{11}=g_{a \gamma} / 10^{-11} \mathrm{GeV}^{-1}$, and $B_{\mu \mathrm{G}}$ the strength of the magnetic field in $\mu \mathrm{G}$. The search for oscillatory features in spectra of active galactic nuclei (AGN) located in or behind galaxy clusters both at X-ray and $\gamma$-ray energies has resulted in stringent limits on the photon-WISP coupling below $m_{\mathrm{neV}} \lesssim 10^{-2}$, and $0.5 \lesssim m_{\mathrm{neV}} \lesssim 100$, respectively (see e.g. Refs. [26, 27]). The narrow spectral features resemble a chaotic pattern of oscillations which depend on the magnetic field properties. Such patterns are not expected in the non-thermal $\gamma$-ray spectra of AGN.

With its wide field of view, AMEGO will observe a plenitude of AGN located in galaxy clusters for which the magnetic fields are often known independently through radio observations. ${ }^{4}$ Assuming the same $B$ field along the line of sight towards the radio galaxy NGC 1275 (the central AGN of the Perseus cluster) as in Ref. [27], the green dashed region in Fig. 3 shows the parameters where the axion or ALP-induced spectral irregularities change the photon flux by at least $\sim 10 \%$ in the AMEGO energy range. With its unprecedented energy resolution of $\sim 1-2 \%$ (Compton events) or $\sim 5 \%$ (pair events) at $10 \mathrm{MeV}$, AMEGO will be perfectly suited to search for these spectral features and bridge the gap in $m_{a}$ not accessible to current X-ray or $\gamma$-ray missions.

At the moment, this mass range is only probed through the non-observation of an ALP-induced $\gamma$-ray burst from SN 1987A [31, 32]. During a core-collapse supernova (SN), ALPs would be produced in the SN core through the conversion of thermal photons in the electrostatic fields of protons and ions. They would quickly escape the core and subsequently convert into $\gamma$-rays in the magnetic field of the Milky Way. This would lead to a short burst lasting tens of seconds that would arrive in coincidence with the SN neutrino burst [33]. The resulting $\gamma$-ray spectrum would have a thermal shape and peak around $\sim 50 \mathrm{MeV}$. No such prompt $\gamma$-ray signal at such high energies is expected otherwise from an $\mathrm{SN}$, making this feature a smoking gun for WISP detection (which falls under category (2) above). The non-observation of such a burst from SN1987A, which occurred in the Large Magellanic Cloud, with the Solar Maximum Mission resulted in a limit of $g_{11} \gtrsim 0.5$ for $m_{\mathrm{neV}} \lesssim 1$ [33]. It has been recently shown that in the event of a Galactic SN observed with the Fermi LAT, and in the absence of the detection of a prompt $\gamma$-ray burst, these limits could be improved by more than an order of magnitude [34]. AMEGO should have a comparable sensitivity to the Fermi-LAT for such an event (shown as a dark green shaded region in Fig. 3): the greatly improved point spread function of $\sim 2^{\circ}$ around $50 \mathrm{MeV}$ compensates for the lower effective area at these energies in comparison to the Fermi-LAT. This entails that AMEGO should be able to probe photon-ALP couplings of $g_{11} \gtrsim 0.02$ for $m_{\mathrm{neV}} \lesssim 1$ and couplings for which ALPs could constitute the dark matter for masses $0.1 \lesssim m_{\mathrm{neV}} \lesssim 10$. It is planned to conduct a dedicated sensitivity study

\footnotetext{
${ }^{3}$ The $\left(m_{a}, g_{a \gamma}\right)$ parameter space for the axion could be greatly enlarged, however, in the case that the axion couples exponentially strong to photons [24].

${ }^{4}$ The knowledge of $B$ fields in galaxy clusters will greatly increase in the future due to the all-sky polarization measurements planned with Square Kilometer Array (SKA) [28].
} 
in order to investigate the full WISP parameter space accessible to AMEGO with a Galactic SN.

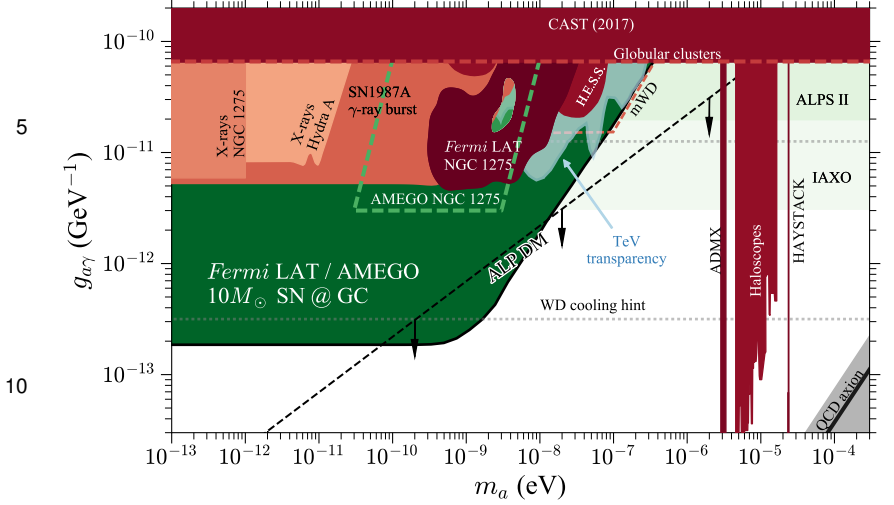

Figure 3: Low mass ALP parameter space. Parameters that will be probed with AMEGO in the case of a Galactic SN are shown as dark green while observations of NGC 1275 should probe parameters within the green dashed lines. Further limits and sensitivities are shown in red and green, respectively (see Ref. [29] and references therein; updated here with the latest CAST bound [30]).

change of the polarization. At $\gamma$-ray energies, the underlying emission mechanism is of non-thermal nature and in case of e.g. synchrotron radiation, an intrinsic polarization is to be expected. Such an intrinsic polarization would have to be modeled in order to extract a WISP effect, which might be feasible for e.g. the Crab Nebula [37].

\section{Summary}

The leading candidates for light dark matter (sub-GeV WIMPs and WISPs) are consistent with all current data and possess distinct $\gamma$-ray signatures. A new instrument like AMEGO, sensitive in the $\mathrm{MeV}$ energy range and with a projected energy and angular resolution that will increase sensitivity by a factor of 20-50 over previous instruments, is now needed in order to properly explore these dark matter candidates. Dark matter searches performed by AMEGO will also be complementary to those carried out by ground-based detectors at much higher energies, thus enabling unprecedented access to its particle nature.

\section{References}

[1] A. Moiseev, All-Sky Medium Energy Gamma-ray Observatory (AMEGO), in 35th International Cosmic Ray Conference, vol. 35 of International Cosmic Ray Conference, p. 8, July, 2017.

[2] J. Racusin and A. Lien, AMEGO: Transients and Multi-Messenger Sources, in 35th International Cosmic Ray Conference, vol. 35 of International Cosmic Ray Conference, p. 8, July, 2017.

[3] J. S. Perkins, M. Ajello, L. Marcotulli, E. Meyer, V. A. Paliya and T. Venters, All-Sky Medium Energy Gamma-ray Observatory (AMEGO), in 35th International Cosmic Ray Conference, vol. 35 of 
[4] G. Steigman, B. Dasgupta and J. F. Beacom, Precise Relic WIMP Abundance and its Impact on Searches for Dark Matter Annihilation, Phys. Rev. D86 (2012) 023506, [1204. 3622].

[5] A. W. McConnachie, The Observed Properties of Dwarf Galaxies in and around the Local Group, AJ 144 (July, 2012) 4, [1204.1562].

[6] FERMI-LAT collaboration, M. Ackermann et al., Searching for Dark Matter Annihilation from Milky Way Dwarf Spheroidal Galaxies with Six Years of Fermi Large Area Telescope Data, Phys. Rev. Lett. 115 (2015) 231301, [1503.02641].

[7] M. Kuhlen, J. Diemand and P. Madau, The Dark Matter Annihilation Signal from Galactic Substructure: Predictions for GLAST, Astrophys. J. 686 (2008) 262, [0 805 . 4416].

[8] M. Pato, F. Iocco and G. Bertone, Dynamical constraints on the dark matter distribution in the Milky Way, JCAP 1512 (2015) 001, [1504.06324].

[9] FERMi-LAT collaboration, M. Ackermann et al., The Fermi Galactic Center GeV Excess and Implications for Dark Matter, Astrophys. J. 840 (2017) 43, [1704. 03910 ].

[10] FERMI-LAT collaboration, M. Ajello et al., Characterizing the population of pulsars in the Galactic bulge with the Fermi Large Area Telescope, Submitted to: Astrophys. J. (2017) , [1705. 00009 ].

[11] E. Charles, M. Sánchez-Conde, B. Anderson, R. Caputo, A. Cuoco, M. Di Mauro et al., Sensitivity projections for dark matter searches with the Fermi large area telescope, Phys. Rep. 636 (June, 2016) 1-46, [1605.02016].

[12] N. Arkani-Hamed, D. P. Finkbeiner, T. R. Slatyer and N. Weiner, A Theory of Dark Matter, Phys. Rev. D79 (2009) 015014, [0810.0713].

[13] M. Raggi and V. Kozhuharov, Results and perspectives in dark photon physics, Riv. Nuovo Cim. 38 (2015) 449-505.

[14] E. Izaguirre, G. Krnjaic, P. Schuster and N. Toro, Analyzing the Discovery Potential for Light Dark Matter, Phys. Rev. Lett. 115 (2015) 251301, [1505. 00011$].$

[15] L. Ackerman, M. R. Buckley, S. M. Carroll and M. Kamionkowski, Dark Matter and Dark Radiation, Phys. Rev. D79 (2009) 023519, [0810.5126].

[16] J. Jaeckel and A. Ringwald, The Low-Energy Frontier of Particle Physics, Ann. Rev. Nucl. Part. Sci. 60 (2010) 405-437, [1 002 . 032 9].

[17] R. D. Peccei and H. R. Quinn, CP conservation in the presence of pseudoparticles, Physical Review Letters 38 (1977) 1440-1443.

[18] S. Weinberg, A new light boson?, Physical Review Letters 40 (1978) 223-226.

[19] F. Wilczek, Problem of strong $P$ and T invariance in the presence of instantons, Physical Review Letters 40 (1978) 279-282.

[20] J. Preskill, M. B. Wise and F. Wilczek, Cosmology of the invisible axion, Physics Letters B 120 (1983) 127-132.

[21] L. F. Abbott and P. Sikivie, A cosmological bound on the invisible axion, Physics Letters B 120 (Jan., 1983) 133-136.

[22] M. Dine and W. Fischler, The not-so-harmless axion, Physics Letters B 120 (Jan., 1983) 137-141.

[23] G. Raffelt and L. Stodolsky, Mixing of the photon with low-mass particles, Phys. Rev. D 37 (1988) 1237-1249. 
[24] M. Farina, D. Pappadopulo, F. Rompineve and A. Tesi, The photo-philic QCD axion, JHEP 01 (2017) 095, [1611.09855].

[25] D. Hooper and P. D. Serpico, Detecting Axionlike Particles with Gamma Ray Telescopes, Physical Review Letters 99 (2007) 231102, [0 706 .3203].

5 [26] D. Wouters and P. Brun, Constraints on Axion-like Particles from X-Ray Observations of the Hydra Galaxy Cluster, ApJ 772 (July, 2013) 44, [1304.0989].

[27] Fermi-LAT collaboration, M. Ajello et al., Search for Spectral Irregularities due to Photon-Axionlike-Particle Oscillations with the Fermi Large Area Telescope, Physical Review Letters 116 (Apr., 2016) 161101.

[28] SKA MAGNETISM WORKING GROUP collaboration, A. Bonafede et al., Unravelling the origin of large-scale magnetic fields in galaxy clusters and beyond through Faraday Rotation Measures with the SKA, PoS AASKA14 (2015) 095, [1501.00321].

[29] FERMI-LAT collaboration, M. Meyer, Searches for Axionlike Particles Using Gamma-Ray Observations, in 12th Patras Workshop on Axions, WIMPs and WISPs (AXION-WIMP 2016) Jeju Island, South Korea, June 20-24, 2016, 2016, 1611.07784.

[30] CAST collaboration, V. Anastassopoulos et al., New CAST Limit on the Axion-Photon Interaction, Nature Phys. 13 (2017) 584-590, [1705. 02290].

[31] J. W. Brockway, E. D. Carlson and G. G. Raffelt, SN 1987A gamma-ray limits on the conversion of pseudoscalars, Physics Letters B 383 (1996) 439-443, [arXiv: astro-ph/9605197].

[32] J. A. Grifols, E. Massó and R. Toldrà, Gamma Rays from SN 1987A due to Pseudoscalar Conversion, Physical Review Letters 77 (1996) 2372-2375, [arXiv: astro-ph/9606028].

[33] A. Payez et al., Revisiting the SN1987A gamma-ray limit on ultralight axion-like particles, J. Cosmology Astropart. Phys. 2 (Feb., 2015) 006, [1410.3747].

[34] M. Meyer, M. Giannotti, A. Mirizzi, J. Conrad and M. Sánchez-Conde, Fermi Large Area Telescope as a Galactic Supernovae Axionscope, Physical Review Letters 118 (2017) 011103, [1609. 02350 ].

[35] D. F. Cowen, A. Franckowiak and M. Kowalski, Estimating the explosion time of core-collapse supernovae from their optical light curves, Astropart. Phys. 33 (Feb., 2010) 19-23, [0901.4877].

[36] A. Lien and B. D. Fields, Cosmic core-collapse supernovae from upcoming sky surveys, J. Cosmology Astropart. Phys. 1 (Jan., 2009) 047, [0 902 .0979].

[37] C. Burrage, A.-C. Davis and D. J. Shaw, Detecting Chameleons: The Astronomical Polarization Produced by Chameleon-like Scalar Fields, Phys. Rev. D79 (2009) 044028, [0 809 . 1763]. 